\title{
Portion size tells who I am, food type tells who you are: Specific functions of amount and type of food in same- and opposite-sex dyadic eating contexts
}

\author{
Nicoletta Cavazza ${ }^{a}{ }^{*}$, Margherita Guidetti ${ }^{a}$, Fabrizio Butera ${ }^{b}$ \\ ${ }^{\mathrm{a}}$ Università di Modena e Reggio Emilia, Italy \\ ${ }^{\mathrm{b}}$ University of Lausanne, Switzerland
}

\section{A R T I C L E I N F O}

\section{Article history:}

Received 3 August 2016

Received in revised form

16 January 2017

Accepted 18 January 2017

Available online 19 January 2017

\section{Keywords:}

Gender identity expression

Ingratiation

Gender-based stereotype about food

Eating behaviour

\begin{abstract}
A B S T R A C T
Previous research has shown that women eating small portions of food (vs. eating big portions) are perceived as more feminine, whereas men eating large portions are perceived as more masculine. The specific type of food items have also been shown to carry connotations for gender stereotyping. In addition, matching the co-eater's food quantity is also a means to ingratiate him or her. Thus, a potential motivational conflict between gender identity expression and ingratiation arises when people eat in opposite-sex dyads. Scholars have, thus far, focused their attention on one of these two dimensions at a time, and rarely in relation to the co-eaters' sex. The present study investigated, through a restaurant scenario, the way in which women and men, when asked to imagine having lunch in dyads, combine food choice and quantity regulation as a function of the co-eater's sex. Results showed that participants use the quantity dimension to communicate gender identity, and the food type dimension to ingratiate the co-eater's preferences by matching her/his presumed choice, following gender-based stereotypes about food. In opposite-sex dyads, dishes that incorporate the two dimensions were chosen above the expected frequency.
\end{abstract}

๑) 2017 Elsevier Ltd. All rights reserved.
Mary is sitting at a restaurant table with her friend John. Mary feels she should eat lightly, but at the same time she worries about appearing too picky in front of John. John loves the big burgers with fries that he often eats with his friends, but he is concerned not to appear too uncouth in Mary's eyes. This scenario exemplifies the dilemma people may face when eating in social situations. Indeed, many studies have documented that food choice in social situations fulfils a function of impression management (Herman, Roth, \& Polivy, 2003; Vartanian, 2015). In particular, this literature shows that both the quantity of food eaten and the type of food chosen are useful tools in signaling gender identity and creating a good impression in co-eaters' eyes (for a review, see Vartanian, Herman, \& Polivy, 2007). This is because there are cultural shared expectations regarding how much and what kind of food a feminine woman or a "real man" should eat: For example, women are expected to prefer vegetables, white meat, fish or dairy food in small

\footnotetext{
* Corresponding author. Università di Modena-Reggio Emilia, via Allegri 9, 42121 Reggio Emilia, Italy.

E-mail address: nicoletta.cavazza@unimore.it (N. Cavazza).
}

quantities, whereas men are expected to prefer large portions of red meat (e.g. Rozin, Hormes, Faith, \& Wansink, 2012; Stein \& Nemeroff, 1995). Thus, when people conform to those expectations they successfully appear as particularly feminine or masculine (for a recent review, see Higgs \& Thomas, 2016).

However, another line of research examining impression management revealed that adapting one's behaviour to that of a coeater is also a means by which to gain social approval (for a recent meta-analysis, see Vartanian, Spanos, Herman, \& Polivy, 2015). Indeed, individuals eating in pairs tend to adapt their food intake to that of their companion in order to enhance social acceptance (e.g. Hermans et al., 2012; Robinson, Tobias, Shaw, Freeman, \& Higgs, 2011). Therefore, in same-sex dyads, eating the amount and the type of food stereotypically associated with one's gender would be the best choice, as this allows to express one's own gender identity while at the same time matching the other's presumed preferences (on the basis of gender stereotypes). But in opposite-sex dyads, the motivation to express one's own gender identity while also ingratiating the co-eater via imitation could diverge and pose a problem of choice.

To date, scholars interested in expressive and communicative 
functions of food have mostly studied the quantity and quality dimensions of food choice regulation separately (e.g. Dibb-Smith and Brindal, 2015; Hermans et al., 2012; Kaisari and Higgs, 2015). A recent exception is a study in which both portion size and food type (masculine vs feminine) were manipulated and crossed. It showed that they both influenced the perceived food-gender association and that this association mediated the intention of women and men to eat their gender-congruent food, irrespective of the situation in which the dish will be consumed (Cavazza, Butera, \& Guidetti, 2015a). However, in this study participants evaluated their intention to eat the proposed foods without reference to a concrete interpersonal context. Thus, only the function of gender identity expression has been considered, while neglecting the ingratiating motivation.

We present a study in which we investigated whether people eating in dyads, and in conditions in which they decide both the amount and the type of food to be eaten, ascribe a specific function to quantity and quality dimensions in order to manage both their gender identity expression and the presumed preferences of the coeater, based on self- and other-stereotypes, respectively.

\section{Food amount}

In the epic 20th century movie Gone with the wind, the iconic female lead Scarlett is advised by Mammy to eat like a bird when it comes to having dinner with Mr Wilkes, as befits a lady. That food amount suppression could be a matter of femininity display is a piece of folk wisdom that received empirical support in scientific literature. Indeed, different studies documented that eating little elicits a feminine impression (Chaiken and Pliner, 1987; Pliner and Chaiken, 1990). The association between eating lightly, or dieting, and femininity is widespread and shared (Bourdieu, 1984; Fagerli and Wandel, 1999; McPhail, Beagan, \& Chapman, 2012; Sobal, 2005). Moreover, limiting the food amount seems to be a deliberate behaviour for women, who were shown to regulate food consumption as a strategy of gender identity reaffirmation (Mori, Chaiken, \& Pliner, 1987; Robinson et al., 2011). In line with this, Young, Mizzau, Mai, Sirisegaram, and Wilson (2009) observed male and female university students eating together in the naturalistic setting of a cafeteria in Canada. They confirmed the main effect of eater's sex, such that women tended to eat less than men. However, interestingly, they also highlighted that, in same-sex dyads, men and women ate a similar amount of calories, whereas in opposite sex-dyads, women particularly tended to choose food with fewer calories than men. In sum, if eating lightly is a behaviour particularly manifested by women, they further minimize their intake when motivated to affirm their own gender identity (e.g., in opposite-sex dyads).

Another line of research highlighted that food quantity may also be regulated in order to match the co-eater's intake, because similarity among co-eaters facilitates the creation of a good impression. Robinson et al. (2011) found that female participants converged with a confederate who ate a large quantity of popcorn after priming need of social acceptance, whereas this convergence did not emerge in a neutral condition.

The two research projects illustrated above exemplify how food amount regulation may fulfil both a function of gender identity expression and of ingratiation, particularly for women. However, in opposite-sex dyads, a woman should eat little in order to appear feminine, but also eat like a man (i.e., a lot) in order to match her coeater. How does she overcome this dilemma? Fortunately, in real situations we have the opportunity to vary another useful symbolic dimension of food: food type.

\section{Food type}

Food type also conveys femininity or masculinity (Sobal, 2005). A great deal of research has examined the so-called gender-stereotyped foods present in every culture (e.g., Counihan and Kaplan, 2004). This line of study showed that red meat is widely perceived as the prototypical food for men, whereas vegetables, dairy products, fish, fruit and sweets are generally considered feminine foods (O'Doherty Jensen \& Holm, 1999). As other gender role expectations, men and women learn in the course of experience what is a gender-appropriate food choice (Rolls, Fedoroff, \& Guthrie, 1991). The consequence is that co-eaters perceive masculinity or femininity of targets based on the foods they eat (e.g., Stein and Nemeroff, 1995). Observers rate both men and women eating "feminine" foods as more feminine than those eating "masculine" foods (Chaiken and Pliner, 1987; Mooney and Lorenz, 1997; Mori et al., 1987; Stein and Nemeroff, 1995).

Likewise, White and Dahl (2006) found that food choices are influenced by the desire of the eaters to dissociate themselves from a devaluated reference group (e.g., men preferred not to eat a steak that was defined in the menu as a "lady's cut"). Gal and Wilkie (2010) proposed a similar manipulation of the dish label, naming the same courses in a menu either in a feminine (e.g., Filet Paulette) or in a masculine way (e.g., Rutherford Ribeye). Participants had to order a meal in conditions of high vs low cognitive resource availability. Results showed that women tended to choose a greater number of feminine than masculine items, irrespective of resource availability, whereas men tended to order gender congruent dishes particularly in a condition of high resource availability.

However, these studies did not consider the co-eaters' sex. Actually, research examining gender-stereotypical food consumption has almost exclusively limited the attention to food's expressive function of identity, whereas evidence regarding the influence of a co-eater's characteristics is very scant. Taking the co-eaters' sex into consideration, a recent study (Cavazza, Guidetti, \& Butera, 2015b) showed that participants tended to prefer their co-eaters' gender-congruent foods over and above their own gendercongruent foods. In this case participants had to express the likelihood to eat either a Caprese salad (i.e., a feminine dish) or a hamburger (i.e., a masculine dish) during a dinner with a men or a women. Male and female participants were more likely to prefer the feminine food when eating with women and the less feminine food when eating with men, even though this orientation to adapt their choice to the co-eaters was particularly true for men. This pattern did not change when the co-eater was a dating partner. However, participants only had the opportunity to vary their choice of food type, but not the amount, as a function of their co-eaters' sex.

\section{The present research}

Examining the literature on the psychological functions covered by food regulation and food choice in terms of gender identity expression and ingratiation, a motivational conflict appears as a potential outcome of eating in opposite-sex dyads. Indeed, when a person eats with an opposite-sex partner, the motivation to model the presumed other's choice is at odds with the motivation to express one's own gender identity (i.e. making gender-congruent choices). The question of whether individuals reconcile the two motivations, ascribing a specific and prevalent function to quantity regulation and food choice, is still open, because the experimental paradigms used in the studies reviewed above did not give participants the opportunity to vary both food type and amount in relation to the co-eater's sex. This is why we devised the present study, in which we asked male and female participants to imagine 
having lunch with a (female vs. male) friend and asked them to choose one dish from a fictitious menu including a list of male- and female-stereotyped dishes, either in medium or big portions.

On the basis of the literature reviewed above, we could infer that the regulation of food amount and the choice of food type may be strategically used in order to, respectively, behave in a gendercongruent way and model the partner at the same time. This would allow the eater to satisfy two different expressive functions of food - that is, conveying one's own gender identity while ingratiating the co-eater.

More specifically, we formulate the following hypotheses:

Hypothesis 1. On the basis of the findings by Young et al. (2009), we expected the quantity dimension to be used for the gender identity expressive function. In operational terms, we expected to find more frequent gender-congruent than -incongruent choices on the quantitative dimension (i.e. medium portions, and dishes estimated as less caloric, for women, vs. big portions, and dishes estimated as more caloric, for men).

Hypothesis 2. In line with the findings by Cavazza et al. (2015b), we expected the food choice dimension to be used for the ingratiating function. In operational terms, we expected to observe more frequent partner's gender-congruent than -incongruent choices on the food type (i.e. feminine dishes for people lunching with women and masculine dishes for people lunching with men).

Hypothesis 3. Accordingly, as a way to satisfy both the motivation of gender identity expression and co-eater ingratiation, we expected to find a combination of the choices of food amount and food type as a function of the dyad composition. In particular, we should find over-representation of medium-portion masculine dishes among women in dyads with men, and of big portions of feminine dishes among men in dyads with women. In contrast, we should observe an over-representation of medium portions of feminine dishes among women in dyads with other women, and big portions of masculine dishes among men in dyads with other men.

\section{Method}

The purpose of the present study was to explore the combination of quantity and type of food in the choice of men and women as a function of the co-eater's sex. In order to test the hypotheses, the experiment design was a 2 (respondents' sex) x 2 (co-eater's sex) between-participants, and the menu list was set in order to offer two categories of dish type (masculine and feminine), each in two portion sizes (medium or big).

\subsection{Participants}

One hundred and eighty-eight participants (59\% women) aged $18-65(M=28.55, S D=9.96)$ were recruited through a university student mailing list, Facebook contacts and snowball sampling. They gave their informed consent to participate on a voluntary basis in the study and were asked to complete an online questionnaire about food habits implemented on the LimeSurvey platform.

\subsection{Design and procedure}

The questionnaire presented a restaurant scenario in which participants were invited to imagine having lunch with a female or male friend. Participants were randomly distributed to the male/ female co-eater condition. In order to make salient the manipulation of the partner's sex, we asked participants to think of an actual friend and write down his/her first name. In case of incongruence between the assigned condition and the name written by participants $(n=39)$, we assigned the participants to the condition that corresponded to the name they wrote. They were then invited to read the menu and choose only one dish - the one they would like to eat in that situation - along with the portion size. The menu prepared for experimental purposes included 12 options, each of them available in a medium or a big portion. It should be noted that we offered medium and big portions, instead of small and big, because we limited the choice to one item, and choosing just a small portion of a single dish would not be enough in size to constitute a lunch to the participants.

The selection of the dishes was made on the basis of a pilot study in which 30 participants had to categorize a list of 26 prepared dishes according to how much they associated these dishes with men, women, or neither. We included in the experimental menu the six dishes associated with men and the six associated with women by at least $70 \%$ of the pilot respondents. All dishes associated with men included pork or red meat (e.g., pork knuckles in spicy sauce), whereas the dishes associated with women included chicken, vegetables and/or cheese (e.g., caprese salad). The scenario did not include a decision about wine or other beverages to drink with food. No actual foods were presented to participants. The gendered stereotypicality of the dish (feminine vs. masculine) and the portion size (medium vs big) were our critical dependent variables.

Before reading the menu, the participants were asked to report how hungry they felt on a 10 -point scale (from $1=$ not at all, to 10 = very much).

After having stated their choices, participants were invited to estimate the calories of the chosen dish. Then, the questionnaire included some personality scales that were not used for the present purposes. ${ }^{1}$ Finally, participants were invited to report some sociodemographic information. The goal of the study was then explained, and respondents were thanked for their participation.

\section{Results}

In the present study, we expected to find systematic variations of food type choice and quantity regulation as a function of the dyad composition (same- vs opposite sex), as evidence of the need to combine both the motivation to express one's gender identity and to ingratiate the co-eater.

Globally, 39\% of the participants chose a medium portion of a masculine dish, $26.7 \%$ a medium portion of a feminine dish, $23.5 \%$ a big portion of a masculine dish, and $10.7 \%$ a big portion of a feminine dish. In order to test Hypotheses 1 and 2, logistic regression analyses with backward stepwise elimination were performed. This analysis begins with the test of the full model, as in ordinary linear regressions, and sequentially removes terms. At each stage, it removes the least damaging term for the model (e.g., that with the largest $p$-value). The process stops when any further deletion significantly decreases the model fit (Agresti \& Kateri, 2011).

We considered a first logistic regression model, where portion size and sex of the respondent, sex of the co-eater, the interaction term, and hunger evaluation (control variable) were the predictors,

\footnotetext{
1 The personality scales included in the questionnaire were the Ingratiation and Self-promotion subscales from the Bolino and Turnley (1999) ImpressionManagement Scale; the short version (Bobbio \& Manganelli, 2011) of Paulhus (1988) Balanced Inventory of Desirable Responding (BIDR 6); the Self-Monitoring Scale (Snyder, 1974) in the Italian version validated by Delle Grazie (2008). A series of preliminary analyses revealed that any of these construct interacted with our independent variables.
} 
as the starting point of the stepwise backward elimination model selection procedure. In line with Hypothesis 1 , the final model, $\chi^{2}$ (1) $=7.29, p=0.007,-2 L L=233.01$, Cox and Snell $R^{2}=0.04$, Nagelkerke $R^{2}=0.05$, included the significant effect of the respondents' sex, $b=-0.84, E S=0.31, \operatorname{Exp}(b)=0.43, p=0.007$. Women chose a medium portion and men chose a big portion more than the expected value (Adj. Res. $= \pm 2.7$ ), irrespective of the coeater's sex (Table 1).

In addition, an analysis of variance on the calorie estimate of the dish chosen as a function of sex of the respondent and sex of the coeater confirmed that women reported a lower estimate of calories for the dish chosen $(M=349.01, S D=160.65)$ than men $(M=424.50, S D=204.11), F(1,155)=6.90, p=0.009, \eta^{2}=0.04$, irrespective of the co-eater's sex and after controlling for the hunger effect.

A second identical logistic regression model was considered with gendered stereotypicality of the dish as the dependent variable $\left(0=\right.$ masculine, $1=$ feminine dish). The final model, $\chi^{2}$ $(2)=9.16, p=0.03,-2 L L=240.09$, Cox and Snell $R^{2}=0.05$, Nagelkerke $R^{2}=0.06$, included the significant effect of the coeater's sex, $b=-0.74, E S=0.33, \operatorname{Exp}(b)=0.48, p=0.024$, after controlling for hunger effect, $b=-0.12, E S=0.06, \operatorname{Exp}(b)=0.89$, $p=0.046$. When imagining having lunch with a female friend, the choice of a feminine dish was significantly more frequent than expected, whereas masculine dishes were chosen more than expected when participants imagined having lunch with a male friend (Adj. Res. $= \pm 2.2$ ), irrespective of a participant's sex (Table 2).

In order to capture the combined use of food-amount and foodtype dimensions as a strategic way of satisfying the two motivations of gender identity expression and co-eater ingratiation (H3), we analyzed the distribution of the chosen dish category (masculine/big portion, masculine/medium portion, feminine/big portion, feminine/medium portion) as a function of dyad composition (same sex, opposite sex). The chi-squared analyses confirmed that the dish choice was influenced by the dyad composition. For the same-sex dyads, the relationship was only marginally significant, $\chi^{2}(3)=6.34, p=0.096$; however, male respondents in same-sex dyads tended to choose big portions of masculine dishes more than expected, and conversely, female respondents tended to avoid this same kind of choice (Adj. Res. $= \pm 2.4$; Table 3). For opposite-sex dyads, in line with our hypothesis, men chose a big portion of a feminine dish more than expected (Adj. Res. $=2.2$ ), and women chose a medium portion of a masculine dish more than expected (Adj. Res. $=2.6), \chi^{2}(3)=10.02, p=0.018$ (Table 3 ).

\section{Discussion}

Our findings confirmed that the sex composition of a dyad at a table actually differentially affects the choice of food type and the regulation of food amount. With the present study we intended to bridge two usually separated literature: that concerning the psychological functions of food amount regulation and that concerning

Table 1

Observed and expected frequencies of choice of portion size as a function of the participants' sex.

\begin{tabular}{clll}
\hline & Medium Portion & Big Portion & Total \\
\hline Women & & & \\
Obs. & 81 & 29 & 110 \\
Exp. & 72.4 & 37.6 & \\
Men & & & \\
Obs. & 42 & 35 & 77 \\
Exp. & 50.6 & 26.4 & 187 \\
Total & 123 & 64 &
\end{tabular}

Table 2

Observed and expected frequencies of choice of a gendered stereotypical dish as a function of the co-eater's sex.

\begin{tabular}{llll}
\hline & Feminine Dish & Masculine Dish & Total \\
\hline Female co-eater & & & \\
$\quad$ Obs. & 51 & 65 & 116 \\
Exp. & 43.8 & 72.2 & \\
Male co-eater & & & \\
Obs. & 20 & 52 & 72 \\
$\quad$ Exp. & 27.2 & 44.8 & 188 \\
Total & 71 & 117 & \\
\hline
\end{tabular}

food type choice. Previous research has shown that both food choice and amount regulation can be tools with which the eaters convey their gender identity and manage impressions given to others. However, scholars, thus far, have focused their attention on one of these two dimensions at a time, and rarely in relation to the co-eaters' sex.

Our findings clearly showed that, when the opportunity to decide what and how much to eat is available, as generally happens in real dining situations, our participants signaled their gender identity mainly through food amount regulation (women by choosing medium, less caloric portions, and men by choosing big, more caloric portions), which is in line with Hypothesis 1. At the same time, participants also considered the specific otherstereotype: they ingratiated the co-eater mainly by taking advantage of the gendered stereotypicality of food and matching the presumed partner's preference (masculine dishes were preferred with men and feminine dishes with women), which is in line with Hypothesis 2. Importantly, this functional specificity of food amount and food type allowed prospective eaters to reconcile the motivational conflict potentially arising in opposite-sex dyads. Indeed, as expected ( $\mathrm{H} 3)$, a tendency to converge on dishes that incorporate the two dimensions emerged in this condition, which resulted in the choice of the dishes that synthesize the possibility of both ingratiating the co-eater, thanks to the gender stereotypicality of the food, and communicating their own gender identity with the portion size.

Because impression management concerns are served by fulfilling social expectations (Leary and Kowalski, 1990), and in line with studies showing that social expectations are more binding for women than for men, particularly in the food domain (Chaiken and Pliner, 1987; Pliner and Chaiken, 1990; Graziani, Cavazza, \& Guidetti, 2017), we observed that the medium portion of feminine dishes was the most chosen category among our female participants ( 23 out of 42 ), whereas men choosing a big portion of a feminine dish when in feminine company, although higher than the expected frequency, remained a minority (5 out of 47). About onethird of men imagining having lunch with a woman preferred to make a totally gender-congruent choice (i.e. a big portion of masculine food).

The functional specificity of quantity regulation and food choice was also confirmed by the over-representation of the choice of totally gender-congruent dishes in same-sex dyads. In such a condition, male participants were particularly prone to make a "real men" choice (i.e. big portion of meat-based dishes). This is in line with previous findings highlighting the avoidance of feminine foods by men as a means by which to dissociate from the outgroup (Gal \& Wilkie, 2010; White \& Dahl, 2006); however, the present results go one step further in showing that this is more likely in a gender-homogeneous context. In a gender-mixed context, a need to converge with the lunch companion pushes some men towards more feminine (and light) foods. This suggests that the functional specificity attributed to food-amount regulation and choice we 
Table 3

Observed and expected frequencies of dish category chosen as a function of dyad composition.

\begin{tabular}{|c|c|c|c|c|c|}
\hline & Feminine Medium Portion & Feminine Big Portion & Masculine Medium Portion & Masculine Big Portion & Total \\
\hline \multicolumn{6}{|c|}{ Same sex dyads } \\
\hline \multicolumn{6}{|l|}{ Men } \\
\hline Obs. & 5 & 4 & 10 & 11 & \multirow[t]{2}{*}{30} \\
\hline Exp. & 7.7 & 4.6 & 11.3 & 6.4 & \\
\hline \multicolumn{6}{|c|}{ Women } \\
\hline Obs. & 20 & 11 & 27 & 10 & \multirow[t]{2}{*}{68} \\
\hline Exp. & 17.3 & 10.4 & 25.7 & 14.6 & \\
\hline \multicolumn{6}{|c|}{ Opposite sex dyads } \\
\hline \multicolumn{6}{|c|}{ Men } \\
\hline Obs. & 14 & 5 & 13 & 15 & \multirow[t]{2}{*}{47} \\
\hline Exp. & 13.2 & 2.6 & 19.0 & 12.1 & \\
\hline \multicolumn{6}{|c|}{ Women } \\
\hline Obs. & 11 & 0 & 23 & 8 & \multirow[t]{2}{*}{42} \\
\hline Exp. & 11.8 & 2.4 & 17.0 & 10.9 & \\
\hline Total & 50 & 20 & 73 & 44 & 187 \\
\hline
\end{tabular}

observed may have a secondary benefit in promoting food variety. We consider this an avenue worth exploring.

Of course, we recognize that the proposed fictitious scenario only partially represents what happens in real situations where the dish has to be actually eaten after the choice. First of all, our participants did not have to worry about the dinner cost, whereas in real situation this could be part of the selection criteria set. Participants' BMI, physical activity and socio-economic status may also influence their choice of a meal dimension and energy content (e.g. Liebman et al., 2003; Sato, Gittelsohn, Unsain, Roble, \& Scagliusi, 2016), and we did not control for these variables in the current study. Thus, the applicability of these results may need additional investigation because they were derived from choices of hypothetical dishes under artificial and simplified conditions. Future studies might overcome this limitation through the observation of actual choices in restaurants.

Furthermore, we did not take into account the nature of the relationship between the co-eaters, and thus we cannot conclude at this time as to whether contexts of simple acquaintance, friendship or romantic relationship induce variations in the combination of food-amount and food-type dimensions due to the variation in the urge to manifest one's own gender appropriateness and impression management concerns. However, since people are particularly motivated to impress others when they are strangers than when they are friends, we might even expect that results would have been much stronger had we evoked a simple acquaintance or a potential romantic partner rather than a friend as (hypothetical) co-eater. Investigating the exact role for the nature of the interpersonal relationship is left to future research.

Another challenge in this context is the question of whether the strategy of choosing a dish according to the co-eater's sex and regulating the portion size according to one's own gender expectations is actually successful in both communicating gender identity and making a good impression on others. We speculate that the attribution of specific functions to food type and food amount is due to the fact that individuals have learned from experience that it works. However, future research could manipulate the combination of food amount and food type chosen by a fictitious (male or female) target in order to compare impressions given to men and women potentially eating with him/her, and also deepen our understanding of whether people are aware of these functioning.

\section{Conclusion}

Notwithstanding these limitations, the present research is the first (to the best of our knowledge) to show that, when eating with a partner, people regulate both the food type and the food amount with a view to expressing their gender identity and their impression management needs, respectively. In other words, individuals appear to use the quantity dimension to communicate gender identity, and the food type dimension in order to ingratiate the coeater by matching her/his presumed choice, in line with the gender-based stereotypes about foods. In this respect, this study contributes to the understanding of the processes by which individuals deal with their food choices by showing the importance of taking into account the conflicting psychological and normative forces involved in these choices.

\section{Acknowledgements}

We wish to express our gratitude to Elisabetta Brombin for collecting the data and Alberto Roverato for statistical advices.

\section{References}

Agresti, A., \& Kateri, M. (2011). Categorical data analysis. Berlin Heidelberg: Springer. Bobbio, A. N. D. R. E. A., \& Manganelli, A. M. (2011). Measuring social desirability responding. A short version of Paulhus' BIDR 6. Testing, Psychometrics Methodology in Applied Psychology, 18, 117-135.

Bolino, M. C., \& Turnley, W. H. (2003). More than one way to make an impression: Exploring profiles of impression management. Journal of Management, 29(2) $141-160$.

Bourdieu, P. (1984). Distinction: A social critique of the judgment of taste. Mass Harvard University Press.

Cavazza, N., Guidetti, M., \& Butera, F. (2015a). Ingredients of gender-based stereotypes about food: Indirect influence of food type, portion size and presentation on gendered intentions to eat. Appetite, 91, 266-272.

Cavazza, N., Guidetti, M., \& Butera, F. (2015b). The gender-based stereotype about food is on the table. Food choice also depends on co-eater's gender. Psicologia Sociale, 10, 161-172.

Chaiken, S., \& Pliner, P. (1987). Women, but not men, are what they eat: The effect of meal size and gender on perceived femininity and masculinity. Personality and Social Psychology Bulletin, 13, 166-176.

Counihan, C. M., \& Kaplan, S. L. (2004). Food and gender: Identity and power. London, UK: Routledge.

Delle Grazie, V. (2008). Italian standardization of the self monitoring scale at 18 items. Psicologia sociale, 3(1), 157-182.

Dibb-Smith, A., \& Brindal, E. (2015). Table for two: The effects of familiarity, sex and gender on food choice in imaginary dining scenarios. Appetite, 95, 492-499.

Fagerli, R. A., \& Wandel, M. (1999). Gender differences in opinions and practices with regard to a "healthy diet". Appetite, 32, 171-190.

Gal, D., \& Wilkie, J. (2010). Real men don't eat quiche: Regulation of genderexpressive choices by men. Social Psychological and Personality Science, 1(4), $291-301$.

Graziani, A. R., Cavazza, N., \& Guidetti, M. (2017). Restoring gender identity when threatened by food consumption: Overeating may influence stereotypical movie choice. Submitted for publication.

Herman, C. P., Roth, D. A., \& Polivy, J. (2003). Effects of the presence of others on 
food intake: A normative interpretation. Psychological bulletin, 129, 873-886.

Hermans, R. C., Lichtwarck-Aschoff, A., Bevelander, K. E., Herman, C. P., Larsen, J. K., \& Engels, R. C. (2012). Mimicry of food intake: The dynamic interplay between eating companions. PLoS One, 7(2), e31027.

Higgs, S., \& Thomas, J. (2016). Social influences on eating. Current Opinion in Behavioral Sciences, 9, 1-6.

Kaisari, P., \& Higgs, S. (2015). Social modelling of food intake. The role of familiarity of the dining partners and food type. Appetite, 86, 19-24.

Leary, M. R., \& Kowalski, R. M. (1990). Impression management: A literature review and two-component model. Psychological Bulletin, 107(1), 34.

Liebman, M., Pelican, S., Moore, S. A., Holmes, B., Wardlaw, M. K., Melcher, L. M., et al. (2003). Dietary intake, eating behavior, and physical activity-related determinants of high body mass index in rural communities in Wyoming, Montana, and Idaho. International journal of obesity, 27(6), 684-692.

McPhail, D., Beagan, B., \& Chapman, G. E. (2012). "I don't want to be sexist but..." Denying and re-inscribing gender through food. Food, Culture \& Society, 15, 473-489.

Mooney, K. M., \& Lorenz, E. (1997). The effects of food and gender on interpersonal perceptions. Sex Roles, 36, 639-653.

Mori, D., Chaiken, S., \& Pliner, P. (1987). "Eating lightly" and the self-presentation of femininity. Journal of Personality and Social Psychology, 53, 693-702.

O'Doherty Jensen, K., \& Holm, L. (1999). Preferences, quantities and concerns: Sociocultural perspectives on the gendered consumption of foods. European Journal of Clinical Nutrition, 53, 351-359.

Paulhus, D. L. (1988). Balanced inventory of desirable responding (BIDR). Acceptance and Commitment Therapy. Measures Package, 41.

Pliner, P., \& Chaiken, S. (1990). Eating, social motives, and self-presentation in women and men. Journal of Experimental and Social Psychology, 26, 240-254.
Robinson, E., Tobias, T., Shaw, L., Freeman, E., \& Higgs, S. (2011). Social matching of food intake and the need for social acceptance. Appetite, 56, 747-752.

Rolls, J. B., Fedoroff, C. I., \& Guthrie, F. J. (1991). Gender differences in eating behavior and body weight regulation. Health Psychology, 10, 133-142.

Rozin, P., Hormes, J. M., Faith, M. S., \& Wansink, B. (2012). Is meat male? A quantitative multimethod framework to establish metaphoric relationships. Journal of Consumer Research, 39, 629-643.

Sato, P. D. M., Gittelsohn, J., Unsain, R. F., Roble, O. J., \& Scagliusi, F. B. (2016). The use of Pierre Bourdieu's distinction concepts in scientific articles studying food and eating: A narrative review. Appetite, 96, 174-186.

Snyder, M. (1974). Self-monitoring of expressive behavior. Journal of Personality and Social Psychology, 30(4), 526-537.

Sobal, J. (2005). Men, meat, and marriage: Models of masculinity. Food and Foodways, $13,135-158$

Stein, R. I., \& Nemeroff, C. J. (1995). Moral overtones of food: Judgments of others based on what they eat Personality and Social Psychology Bulletin, 21, 480-490.

Vartanian, L. R. (2015). Impression management and food intake. Current directions in research. Appetite, 86, 74-80.

Vartanian, L. R., Herman, C. P., \& Polivy, J. (2007). Consumption stereotypes and impression management: How you are what you eat. Appetite, 48, 265-277.

Vartanian, L. R., Spanos, S., Herman, C. P., \& Polivy, J. (2015). Modeling of food intake: A meta-analytic review. Social Influence, 10, 119-136.

White, K., \& Dahl, D. W. (2006). To be or not be: The influence of dissociative reference groups on consumer preferences. Journal of Consumer Psychology, 16, 404-413.

Young, M. E., Mizzau, M., Mai, N. T., Sirisegaram, A., \& Wilson, M. (2009). Food for thought. What you eat depends on your sex and eating companions. Appetite, 53, 268-271. 